\title{
FILLING IN THE GAPS IN THE 4.85 GHz SKY
}

\author{
Stephen E. Healey ${ }^{1}$, Lars Fuhrmann ${ }^{2}$, Gregory B. Taylor ${ }^{3,5}$, Roger W. Romani ${ }^{1}$, and Anthony C. S. Readhead ${ }^{4}$ \\ ${ }^{1}$ Department of Physics/KIPAC, Stanford University, Stanford, CA 94305, USA; sehealey@astro.stanford.edu \\ ${ }^{2}$ Max-Planck-Institut für Radioastronomie, Bonn, Germany \\ ${ }^{3}$ Department of Physics and Astronomy, University of New Mexico, Albuquerque, NM 87131, USA \\ ${ }^{4}$ Department of Astronomy, California Institute of Technology, Pasadena, CA 91125, USA \\ Received 2009 April 7; accepted 2009 July 2; published 2009 August 26
}

\begin{abstract}
We describe a $4.85 \mathrm{GHz}$ survey of bright, flat-spectrum radio sources conducted with the Effelsberg $100 \mathrm{~m}$ telescope in an attempt to improve the completeness of existing surveys, such as CRATES. We report the results of these observations and of follow-up $8.4 \mathrm{GHz}$ observations with the VLA of a subset of the sample. We comment on the connection to the Wilkinson Microwave Anisotropy Probe point source catalog and on the survey's effectiveness at supplementing the CRATES sky coverage.
\end{abstract}

Key words: galaxies: active - quasars: general - radio continuum: galaxies - surveys

Online-only material: machine-readable and VO tables

\section{INTRODUCTION}

Extensive effort has been made to survey the sky at $4.85 \mathrm{GHz}$ with single-dish telescopes. The Parkes-MIT-NRAO survey (PMN; Griffith \& Wright 1993), conducted with the $64 \mathrm{~m}$ telescope at Parkes, covers the region $-87^{\circ}<\delta<+10^{\circ}$ to a flux density limit of $\sim 30 \mathrm{mJy}$. The Green Bank $6 \mathrm{~cm}$ survey (GB6; Gregory et al. 1996), conducted with the former $91 \mathrm{~m}$ telescope at Green Bank, covers the region $0^{\circ}<\delta<+75^{\circ}$ to a limit of $\sim 18 \mathrm{mJy}$. The Fifth $5 \mathrm{GHz}$ Strong Source Survey (S5; Kühr et al. 1981), conducted with the Effelsberg $100 \mathrm{~m}$ telescope, covers the region $+70^{\circ}<\delta<+90^{\circ}$ to a limit of $\sim 250$ mJy. Together, they constitute a (non-uniform) catalog of almost 125,000 distinct radio sources over nearly the entire sky.

Data from these surveys were subsequently used to select targets for more specialized study. The Cosmic Lens All-Sky Survey (CLASS; Myers et al. 2003; Browne et al. 2003) identified sources that were bright in GB6 and had flat spectra between the 1.4 GHz NRAO VLA Sky Survey (NVSS; Condon et al. 1998) and GB6; these were then observed at $8.4 \mathrm{GHz}$ with the VLA to look for gravitationally lensed compact radio sources. Although the objective of CLASS was to find lenses, Sowards-Emmerd et al. (2003) demonstrated its usefulness in identifying $\gamma$-ray blazars, which correlate strongly with bright, flat-spectrum radio sources. The Combined Radio All-sky Targeted Eight GHz Survey (CRATES; Healey et al. 2007) extended the CLASS procedure to the entire sky with the explicit goal of identifying blazar candidates. It used the full complement of $4.85 \mathrm{GHz}$ sources to identify over 11,000 bright sources with flat spectra, which were followed up with $8.4 \mathrm{GHz}$ interferometry.

Much of the utility of the CRATES sample, especially with regard to identifying blazar candidates, is in its breadth of coverage and its uniformity. Sowards-Emmerd et al. (2003), for example, developed a figure of merit for associating radio sources with $\gamma$-ray detections. The ability to do so in a statistically meaningful way rested on the uniform selection criteria and large area coverage of CLASS. Similarly, the Candidate Gamma-Ray Blazar Survey (CGRaBS; Healey et al. 2008) is a catalog of the $\sim 15 \%$ of CRATES sources that are most similar to the Third EGRET Catalogue (3EG; Hartman

\footnotetext{
5 G. B. Taylor is also an Adjunct Astronomer at the National Radio Astronomy Observatory.
}

et al. 1999) blazars as quantified by a radio/X-ray figure of merit. Without the large number of consistently selected sources provided by CRATES, a catalog with the statistical power of CGRaBS would be impossible to compile. Improving the sample's coverage is further motivated by comparison with other all-sky surveys, such as the Wilkinson Microwave Anisotropy Probe (WMAP) five-year point source catalog (Wright et al. 2008). This shallow but uniform high-frequency radio survey of the entire sky is composed mostly of bright, flat-spectrum sources; thus, the identification of low-frequency counterparts depends on the availability of all-sky low-frequency data.

It is therefore of some importance to note that the sky coverage of the large $4.85 \mathrm{GHz}$ surveys-S5, GB6, and PMN-is not perfect. In the north polar cap (an area of $\sim 700 \mathrm{deg}^{2}$ ), the S5 survey is considerably shallower than GB6 and PMN, as noted above. There are also two "holes" in the PMN survey just south of the equator (an area of $\sim 300 \mathrm{deg}^{2}$ ) in which no data are available at all. These holes are already known to contain $\gamma$-ray blazars in 3EG and in the list of bright active galactic nuclei (Abdo et al. 2009) detected in the first three months of data from the Large Area Telescope (LAT) on board the Fermi Gamma-ray Space Telescope. Likewise, it is expected that $\gamma$-ray blazars not associated with S5 sources will also be detected by the LAT in the far north. The real solution to this problem is a full $4.85 \mathrm{GHz}$ survey of the entire northern cap and PMN hole regions down to the CRATES flux density limit $(\sim 65 \mathrm{mJy})$ or better; such a campaign would require approximately a week of observing time. However, in the interest of time and logistics, we have conducted a targeted $4.85 \mathrm{GHz}$ survey, requiring only $\sim 24 \mathrm{hr}$ of observing time, of selected sources in these regions with the Effelsberg $100 \mathrm{~m}$ telescope as an initial step toward filling in the gaps in the CRATES all-sky coverage.

\section{SAMPLE SELECTION}

\subsection{Sky Regions}

Our work concerns two distinct regions on the sky. The "north polar cap" is the region $+75^{\circ}<\delta<+90^{\circ}$, spanning all right ascensions. The "PMN holes" are two nearly parallelogramshaped regions just south of the equator. Their vertices, given as $(\alpha, \delta)$ in decimal degrees (J2000 coordinates), are approximately as follows: 


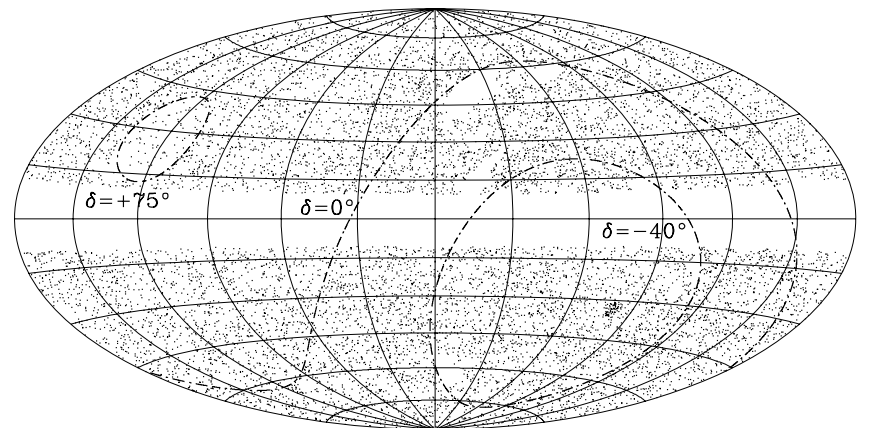

of $\sim 24 \mathrm{hr}$. We used the $4.85 \mathrm{GHz}$ multi-horn receiver permanently mounted at the secondary focus for sensitive continuum measurements. The $4.85 \mathrm{GHz}$ system is a double-horn heterodyne receiver that allows the subtraction of the off-source atmospheric contribution from the on-source astronomical signal. Both circular polarizations are fed into a broadband polarimeter, measuring the total power (Stokes I).

The target sources are sufficiently bright in the observed frequency band to allow flux density measurements using cross-scans in azimuth and elevation. In contrast to the on-off observing technique, this enables an instantaneous control of the telescope pointing as well as the identification of possible problems with confused or extended sources. Sources with $S_{\text {pred }} \geqslant 100 \mathrm{mJy}$ were observed with four cross-scans (two in each direction), and sources with $S_{\text {pred }}<100 \mathrm{mJy}$ were observed with six cross-scans (three in each direction).

In order to minimize slewing time, we observed sources along stripes parallel to the right ascension axis and about $3^{\circ}$ wide in declination. In the case of particularly weak sources (or obviously bad scans), sources were subsequently re-observed to improve $\mathrm{S} / \mathrm{N}$. We observed primary flux density calibrators (e.g., 3C 286, 3C 295, and NGC 7027) frequently to adjust the focus of the telescope and to link the measured flux densities to the absolute flux density scale (Baars et al. 1977; Ott et al. 1994).

The first steps in the data reduction included baseline subtrac-

Figure 1. Top: Aitoff equal-area projection of the CRATES catalog in Galactic coordinates $(l, b)$ with $l=0^{\circ}$ at the center. Bottom: the same sky map with the additional Effelsberg targets shown in red.

PMN hole 1: $\left(175^{\circ}, 0^{\circ}\right) ;\left(183^{\circ}, 0^{\circ}\right) ;\left(197^{\circ},-9^{\circ} .5\right) ;\left(188^{\circ}\right.$, -9.5). PMN hole 2: $\left(195^{\circ}, 0^{\circ}\right)$; $\left(217^{\circ}, 0^{\circ}\right)$; $\left(235^{\circ},-9.5\right)$; $\left(210^{\circ},-9.5\right)$.

\subsection{Choosing Sources}

Our goal was to observe flat-spectrum targets that were likely to be bright at $4.85 \mathrm{GHz}$. In order to select such sources, we drew from archival radio surveys at lower frequencies. NVSS provides $1.4 \mathrm{GHz}$ coverage of the entire $\delta>-40^{\circ}$ sky, but to identify sources with flat spectra, data at a second frequency are required. In the north polar cap, $325 \mathrm{MHz}$ data are available from the Westerbork Northern Sky Survey (WENSS; Rengelink et al. 1997) while in the PMN holes, $365 \mathrm{MHz}$ data are available from the Texas Survey (Douglas et al. 1996). We identified NVSS sources with counterparts in WENSS/Texas and computed the spectral index of each source (assuming a power law spectrum $\left.S \propto v^{\alpha}\right)$. From this, we calculated a predicted $4.85 \mathrm{GHz}$ flux density $\left(S_{\text {pred }}\right)$ for each source. Due to logistical considerations at Effelsberg, the final selection criteria were slightly different for the two observing regions. In the north polar cap, we selected sources with $S_{\text {pred }}>65 \mathrm{mJy}$ and $\alpha>-0.6$ that were not already included in the S5 survey (221 sources). It is worth noting that S5 itself only contains 236 sources, so our observations nearly double the coverage in this region. In the equatorial holes, we selected sources with $S_{\text {pred }}>75 \mathrm{mJy}$ and $\alpha>-1.0(174$ sources).

The top panel of Figure 1 shows a sky map of the sources in the CRATES catalog. The bottom panel shows the additional sources selected for observation at Effelsberg.

\section{OBSERVATIONS AND DATA ANALYSIS}

The flux density measurements of the selected sources were performed with the Effelsberg $100 \mathrm{~m}$ telescope of the MaxPlanck-Institut für Radioastronomie (MPIfR) during two observing runs in 2008 June and July with a total observing time in both slewing directions: the peak of the Gaussian measures the antenna temperature on the source, the HPBW $\left(\sim 144^{\prime \prime}\right)$, and the positional displacement of the peak (i.e., the residual pointing error of the telescope, typically $\left.<10^{\prime \prime}\right)$. We flagged bad scans or subscans and excluded them from further analysis. For sources showing confusion problems (i.e., a secondary source in the off-horn position, causing severe distortion), we reduced the data without the software beam-switch. After correcting the measured amplitudes for residual positional offsets using the approximately Gaussian shape of the telescope beam, we averaged them over both slewing directions. We performed an opacity correction using the $T_{\text {sys }}$ measurements obtained for each subscan and a standard correction for the systematic elevation-dependent telescope gain. The frequent primary calibrator measurements allowed us to convert the measured antenna temperatures for each target into absolute flux densities.

\section{RESULTS}

In total, we observed 395 sources across both sky regions. For 368 (93\%) of the sources, we obtained precise flux densities with a mean fractional uncertainty of $2.3 \%$. The individual measurement uncertainties result mostly from statistical errors in the reduction process (including errors from the Gaussian fit) and a contribution from the scatter seen in the primary calibrator measurements. By independently studying a large sample of weak sources at $4.85 \mathrm{GHz}$, Angelakis et al. (2009) showed that for flux densities $\lesssim 100 \mathrm{mJy}$, the dominant sources of uncertainty are thermal noise, confusion, and tropospheric instabilities. Together, they result in an uncertainty of about $1.2 \mathrm{mJy}$, which we also include in our error estimates. The $4.85 \mathrm{GHz}$ flux densities and uncertainties are given in Table 1 (for the sources in the north polar cap) and Table 2 (for the sources in the PMN holes). The NVSS positions were sufficient given the pointing accuracy of the Effelsberg $100 \mathrm{~m}$ telescope, and they are more accurate than can be measured by the $100 \mathrm{~m}$ telescope, so we report the NVSS positions in the tables. 
Table 1

Sources in the North Polar Cap

\begin{tabular}{|c|c|c|c|c|c|c|c|}
\hline \multirow{2}{*}{$\begin{array}{c}\text { Name } \\
\text { J0010+7614 }\end{array}$} & \multicolumn{2}{|c|}{$\begin{array}{l}\text { NVSS Position } \\
\text { (J2000) }\end{array}$} & \multirow{2}{*}{$\begin{array}{r}\begin{array}{c}S_{4.85} \\
(\mathrm{mJy})\end{array} \\
25.4\end{array}$} & \multirow{2}{*}{$\begin{array}{c}\begin{array}{c}\sigma_{4.85} \\
(\mathrm{mJy})\end{array} \\
1.2\end{array}$} & \multicolumn{2}{|c|}{$\begin{array}{c}\text { VLA 8.4 GHz Position } \\
(\mathrm{J} 2000)\end{array}$} & \multirow[t]{2}{*}{$\begin{array}{l}S_{8.4}{ }^{\mathrm{a}} \\
(\mathrm{mJy})\end{array}$} \\
\hline & $00: 10: 17.83$ & $+76: 14: 19.8$ & & & & & \\
\hline $\mathrm{J} 0022+7501$ & $00: 22: 02.13$ & $+75: 01: 22.5$ & 34.0 & 1.3 & & & \\
\hline J0029+8031 & $00: 29: 27.15$ & $+80: 31: 31.6$ & 80.0 & 1.6 & & & \\
\hline $\mathrm{J} 0032+8750$ & $00: 32: 41.68$ & $+87: 50: 43.7$ & 57.1 & 1.4 & & & \\
\hline $\mathrm{J} 0033+7829$ & $00: 33: 08.23$ & $+78: 29: 00.8$ & 67.4 & 1.5 & $00: 33: 08.211$ & $+78: 29: 00.12$ & $48.5^{\mathrm{b}}$ \\
\hline $\mathrm{J} 0045+8810$ & $00: 45: 02.55$ & $+88: 10: 17.9$ & 66.6 & 1.5 & & & \\
\hline $\mathrm{J} 0054+7558$ & $00: 54: 30.73$ & $+75: 58: 08.0$ & 66.6 & 1.5 & & & \\
\hline J0115+8109 & $01: 15: 42.01$ & $+81: 09: 54.2$ & 52.2 & 1.4 & & & \\
\hline J0128+7928 & 01:28:08.93 & $+79: 28: 46.1$ & 113.3 & 1.9 & 01:28:08.870 & $+79: 28: 46.14$ & 93.0 \\
\hline J0138+8611 & $01: 38: 29.65$ & $+86: 11: 40.1$ & 68.0 & 1.5 & $01: 38: 29.637$ & $+86: 11: 40.90$ & 38.7 \\
\hline $\mathrm{J} 0143+8544$ & $01: 43: 35.52$ & $+85: 44: 22.7$ & 82.0 & 1.6 & $01: 43: 35.551$ & $+85: 44: 23.50$ & 68.4 \\
\hline J0144+7958 & $01: 44: 37.08$ & $+79: 58: 39.5$ & 19.6 & 1.2 & & & \\
\hline $\mathrm{J} 0151+8303$ & $01: 51: 18.31$ & $+83: 03: 20.2$ & 51.6 & 1.4 & & & \\
\hline $\mathrm{J} 0157+7552$ & $01: 57: 11.56$ & $+75: 52: 28.8$ & 102.6 & 1.8 & & & \\
\hline $\mathrm{J} 0202+8115$ & 02:02:05.42 & $+81: 15: 45.0$ & 163.7 & 2.4 & 02:02:05.739 & $+81: 15: 45.28$ & $47.6^{\mathrm{b}}$ \\
\hline $\mathrm{J} 0211+8449$ & $02: 11: 41.58$ & $+84: 49: 47.7$ & 78.4 & 1.6 & $02: 11: 41.338$ & $+84: 49: 47.92$ & 55.5 \\
\hline $\mathrm{J} 0215+7554$ & $02: 15: 17.69$ & $+75: 54: 53.6$ & 96.6 & 1.7 & $02: 15: 17.902$ & $+75: 54: 53.01$ & 68.9 \\
\hline $\mathrm{J} 0249+8019$ & 02:49:40.73 & $+80: 19: 25.3$ & 38.6 & 1.3 & & & \\
\hline $\mathrm{J} 0249+8435$ & 02:49:48.43 & $+84: 35: 56.4$ & 126.7 & 2.0 & $02: 49: 48.331$ & $+84: 35: 57.03$ & 121.0 \\
\hline $\mathrm{J} 0304+7930$ & 03:04:56.81 & $+79: 30: 52.2$ & 26.4 & 1.2 & & & \\
\hline $\mathrm{J} 0309+8601$ & 03:09:42.29 & $+86: 01: 39.7$ & 27.0 & 1.3 & & & \\
\hline $\mathrm{J} 0316+7720$ & $03: 16: 32.19$ & $+77: 20: 59.2$ & 115.6 & 1.9 & $03: 16: 32.143$ & $+77: 20: 58.40$ & 133.7 \\
\hline $\mathrm{J} 0317+7658$ & $03: 17: 51.96$ & $+76: 58: 33.9$ & 83.2 & 1.6 & 03:17:54.072 & $+76: 58: 38.06$ & 13.4 \\
\hline $\mathrm{J} 0320+8711$ & $03: 20: 21.87$ & $+87: 11: 18.6$ & 50.6 & 1.4 & & & \\
\hline J0324+7849 & $03: 24: 14.60$ & $+78: 49: 10.9$ & 106.1 & 1.8 & $03: 24: 14.531$ & $+78: 49: 11.65$ & 82.6 \\
\hline $\mathrm{J} 0325+8517$ & $03: 25: 11.23$ & $+85: 17: 40.0$ & 82.9 & 1.6 & $03: 25: 11.125$ & $+85: 17: 39.45$ & 42.7 \\
\hline & & & & & $03: 25: 13.732$ & $+85: 17: 43.70$ & 4.4 \\
\hline $\mathrm{J} 0410+8208$ & $04: 10: 39.50$ & $+82: 08: 23.3$ & 115.3 & 1.9 & & & \\
\hline $\mathrm{J} 0411+8509$ & $04: 11: 52.15$ & $+85: 09: 43.9$ & 52.5 & 1.4 & & & \\
\hline $\mathrm{J} 0428+7528$ & $04: 28: 18.59$ & $+75: 28: 00.3$ & 18.8 & 1.2 & & & \\
\hline $\mathrm{J} 0428+8314$ & $04: 28: 21.90$ & $+83: 14: 55.3$ & 48.7 & 1.4 & & & \\
\hline $\mathrm{J} 0440+7755$ & 04:40:18.91 & $+77: 55: 58.5$ & 56.3 & 1.4 & & & \\
\hline $\mathrm{J} 0442+8742$ & $04: 42: 25.51$ & $+87: 42: 57.4$ & 21.3 & 1.2 & & & \\
\hline $\mathrm{J} 0449+8233$ & 04:49:00.25 & $+82: 33: 18.8$ & 197.8 & 2.8 & 04:49:00.184 & $+82: 33: 19.45$ & 150.7 \\
\hline $\mathrm{J} 0501+8505$ & 05:01:54.66 & $+85: 05: 19.9$ & 15.3 & 1.2 & & & \\
\hline $\mathrm{J} 0517+8108$ & $05: 17: 57.65$ & $+81: 08: 51.9$ & 13.3 & 1.2 & & & \\
\hline $\mathrm{J} 0524+7627$ & $05: 24: 22.17$ & $+76: 27: 35.4$ & 58.6 & 1.4 & & & \\
\hline $\mathrm{J} 0532+7623$ & $05: 32: 29.80$ & $+76: 23: 28.3$ & 56.8 & 1.4 & & & \\
\hline $\mathrm{J} 0540+7702$ & $05: 40: 52.10$ & $+77: 02: 01.5$ & 46.8 & 1.3 & & & \\
\hline J0546+7943 & $05: 46: 48.52$ & $+79: 43: 25.2$ & 31.1 & 1.3 & & & \\
\hline
\end{tabular}

Notes.

a The typical error in the $8.4 \mathrm{GHz}$ flux density is approximately $3 \%$.

${ }^{\mathrm{b}}$ Component is resolved or partially resolved.

(This table is available in its entirety in machine-readable and Virtual Observatory (VO) forms in the online journal. A portion is shown here for guidance regarding its form and content.)

\section{VLA FOLLOW-UP}

We conducted a follow-up campaign on 109 sources (72 in the north polar cap and 37 in the PMN holes), with the VLA in the A configuration, using two $50 \mathrm{MHz}$ bands at $8.44 \mathrm{GHz}$. We targeted sources with $S_{4.85 \mathrm{GHz}} \geqslant 65 \mathrm{mJy}$ from our Effelsberg observations and $\alpha>-0.6$ between $1.4 \mathrm{GHz}$ (from NVSS) and $4.85 \mathrm{GHz}$. The observations took place on 2008 November 1 as part of program AH0976, and the on-source dwell time was $60 \mathrm{~s}$. Standard AIPS calibration was performed, followed by Difmap imaging and Gaussian component fitting. We used 3C 286 as a flux density calibrator, and the typical radiometric error is approximately $3 \%$. With interferometric $X$-band measurements in hand, these sources are now completely on par with those in CRATES.

The results of these observations are shown in Tables 1 and 2. We report the position and integrated flux density of each source. For sources with multiple components, we report the position and integrated flux density of each individual component. The typical positional accuracy is $\lesssim 0{ }^{\prime} 06$, which is consistent with the positional uncertainties of the calibrators and the expected positional accuracy under good conditions. Four sources (J0033+7829, J0202+8115, J1445-0329, and $\mathrm{J} 2329+7808$ ) have components that were resolved by the VLA; these are less likely to be the compact cores of blazars and are indicated in the tables. 
Table 2

Sources in the PMN Holes

\begin{tabular}{|c|c|c|c|c|c|c|c|}
\hline \multirow{2}{*}{$\begin{array}{c}\text { Name } \\
\text { J1144-0031 }\end{array}$} & \multicolumn{2}{|c|}{$\begin{array}{l}\text { NVSS Position } \\
\text { (J2000) }\end{array}$} & \multirow{2}{*}{$\begin{array}{c}\begin{array}{c}S_{4.85} \\
(\mathrm{mJy})\end{array} \\
215.8\end{array}$} & \multirow{2}{*}{$\begin{array}{c}\begin{array}{c}\sigma_{4.85} \\
(\mathrm{mJy})\end{array} \\
3.1\end{array}$} & \multicolumn{2}{|c|}{$\begin{array}{c}\text { VLA } 8.4 \mathrm{GHz} \text { position } \\
(\mathrm{J} 2000)\end{array}$} & \multirow[t]{2}{*}{$\begin{array}{l}S_{8.4^{\mathrm{a}}} \\
(\mathrm{mJy})\end{array}$} \\
\hline & $11: 44: 54.01$ & $-00: 31: 36.6$ & & & & & \\
\hline J1146-0007 & $11: 46: 40.93$ & $-00: 07: 33.7$ & 42.6 & 1.3 & & & \\
\hline J1146-0108 & $11: 46: 42.88$ & $-01: 08: 05.1$ & 121.8 & 2.0 & & & \\
\hline J1148-0046 & 11:48:07.19 & $-00: 46: 45.3$ & 165.5 & 2.5 & & & \\
\hline $\mathrm{J} 1150-0023$ & $11: 50: 43.88$ & $-00: 23: 54.4$ & 1797.8 & 23.4 & & & \\
\hline $\mathrm{J} 1156-0212$ & 11:56:38.19 & $-02: 12: 53.8$ & 71.8 & 1.5 & & & \\
\hline J1157-0124 & $11: 57: 11.03$ & $-01: 24: 11.0$ & 69.9 & 1.5 & & & \\
\hline $\mathrm{J} 1157-0312$ & $11: 57: 50.08$ & $-03: 12: 23.8$ & 148.2 & 2.3 & & & \\
\hline $\mathrm{J} 1202-0240$ & $12: 02: 32.28$ & $-02: 40: 03.3$ & 219.9 & 3.1 & & & \\
\hline $\mathrm{J} 1202-0336$ & $12: 02: 51.43$ & $-03: 36: 26.6$ & 97.5 & 1.7 & & & \\
\hline J1204-0029 & 12:04:05.69 & $-00: 29: 50.0$ & 157.7 & 2.4 & $12: 04: 05.683$ & $-00: 29: 53.55$ & 50.7 \\
\hline J1204-0422 & $12: 04: 02.13$ & $-04: 22: 44.0$ & 962.7 & 12.6 & & & \\
\hline \multirow[t]{3}{*}{ J1207-0106 } & 12:07:41.66 & $-01: 06: 37.4$ & 153.5 & 2.3 & $12: 07: 41.679$ & $-01: 06: 36.67$ & 107.7 \\
\hline & & & & & $12: 07: 42.244$ & $-01: 06: 47.11$ & 6.5 \\
\hline & & & & & $12: 07: 41.589$ & $-01: 06: 39.67$ & 4.9 \\
\hline J1209-0257 & 12:09:06.88 & $-02: 57: 45.2$ & 106.5 & 1.8 & & & \\
\hline $\mathrm{J} 1210-0136$ & $12: 10: 31.37$ & $-01: 36: 50.1$ & 258.0 & 3.6 & & & \\
\hline $\mathrm{J} 1210-0341$ & $12: 10: 18.86$ & $-03: 41: 54.0$ & 65.4 & 1.5 & & & \\
\hline J1213-0159 & $12: 13: 14.00$ & $-01: 59: 04.0$ & 52.5 & 1.4 & & & \\
\hline J1214-0306 & $12: 14: 50.51$ & $-03: 06: 49.2$ & 86.5 & 1.6 & $12: 14: 50.517$ & $-03: 06: 49.55$ & 65.0 \\
\hline J1214-0416 & $12: 14: 32.37$ & $-04: 16: 03.4$ & 79.6 & 1.6 & & & \\
\hline J1214-0606 & $12: 14: 35.91$ & $-06: 06: 58.9$ & 86.0 & 1.6 & & & \\
\hline $\mathrm{J} 1215-0628$ & $12: 15: 14.42$ & $-06: 28: 03.6$ & 308.0 & 4.2 & $12: 15: 14.394$ & $-06: 28: 03.90$ & 296.9 \\
\hline J1217-0337 & $12: 17: 55.30$ & $-03: 37: 21.2$ & 73.3 & 1.5 & & & \\
\hline $\mathrm{J} 1218-0631$ & $12: 18: 36.18$ & $-06: 31: 15.9$ & 181.4 & 2.6 & & & \\
\hline \multirow[t]{4}{*}{$\mathrm{J} 1221-0241$} & $12: 21: 23.90$ & $-02: 41: 50.9$ & 448.0 & 5.9 & $12: 21: 23.942$ & $-02: 41: 49.60$ & 210.8 \\
\hline & & & & & $12: 21: 23.921$ & $-02: 41: 49.84$ & 31.7 \\
\hline & & & & & $12: 21: 30.395$ & $-02: 41: 32.99$ & 23.0 \\
\hline & & & & & $12: 21: 30.409$ & $-02: 41: 32.95$ & 18.7 \\
\hline J1222-0449 & $12: 22: 24.14$ & $-04: 49: 33.0$ & 62.3 & 1.4 & & & \\
\hline J1226-0421 & $12: 26: 46.60$ & $-04: 21: 18.6$ & 104.2 & 1.8 & & & \\
\hline J1226-0434 & $12: 26: 56.76$ & $-04: 34: 22.2$ & 116.1 & 1.9 & & & \\
\hline $\mathrm{J} 1227-0445$ & $12: 27: 16.53$ & $-04: 45: 32.8$ & 39.5 & 1.3 & & & \\
\hline \multirow[t]{3}{*}{$\mathrm{J} 1228-0631$} & $12: 28: 52.20$ & $-06: 31: 46.3$ & 191.7 & 2.8 & $12: 28: 52.176$ & $-06: 31: 46.55$ & 70.6 \\
\hline & & & & & $12: 28: 52.192$ & $-06: 31: 46.54$ & 29.5 \\
\hline & & & & & $12: 28: 52.209$ & $-06: 31: 46.48$ & 15.6 \\
\hline J1228-0838 & $12: 28: 20.43$ & $-08: 38: 13.1$ & 281.7 & 3.9 & & & \\
\hline $\mathrm{J} 1230-0510$ & $12: 30: 29.00$ & $-05: 10: 01.0$ & 76.0 & 1.6 & & & \\
\hline $\mathrm{J} 1232-0717$ & $12: 32: 52.31$ & $-07: 17: 29.9$ & 172.3 & 2.5 & & & \\
\hline $\mathrm{J} 1233-0613$ & $12: 33: 31.27$ & $-06: 13: 22.8$ & 67.1 & 1.5 & & & \\
\hline
\end{tabular}

Notes.

a The typical error in the $8.4 \mathrm{GHz}$ flux density is approximately $3 \%$.

${ }^{\mathrm{b}}$ Component is resolved or partially resolved.

(This table is available in its entirety in machine-readable and Virtual Observatory (VO) forms in the online journal. A portion is shown here for guidance regarding its form and content.)

\section{WMAP POINT SOURCES}

The WMAP five-year data release includes a list of 390 point sources detected in the sky maps of at least one of the WMAP frequency bands $(K=23 \mathrm{GHz}, K a=33 \mathrm{GHz}, Q=41 \mathrm{GHz}$, $V=61 \mathrm{GHz}, W=94 \mathrm{GHz}$ ). This provides an all-sky highfrequency radio catalog of the sky against which to check our selection of bright, flat-spectrum sources. Healey et al. showed that the vast majority $(\sim 88 \%)$ of sources in the WMAP threeyear point source catalog (Hinshaw et al. 2006) had counterparts in CRATES. Those that did not were dominated by sources that were very bright but had steep spectra. However, some of the sources without counterparts were bright, flat-spectrum sources that were simply located in regions not covered by CRATES (e.g., the Galactic plane and the PMN holes). At the WMAP areal density $\left(9.5 \times 10^{-3} \mathrm{deg}^{-2}\right)$, we expect the PMN holes to contain $\sim 3$ WMAP sources, and indeed, two sources (WMAP J1408-0749 and WMAP J1512-0904) are located there and another (WMAP J1510-0546) is right on the edge of the PMN coverage. Our sample selection included these three sources (i.e., they were not specially targeted), and our $4.85 \mathrm{GHz}$ data confirm that they are indeed bright, flat-spectrum sources like those in the CRATES sample.

\section{DISCUSSION}

Figure 2 shows a comparison of the observed $4.85 \mathrm{GHz}$ flux densities to the flux densities predicted as described in Section 2.2. As the distribution shows, our simple power-law extrapolation is not a very good indicator of the true flux density; it overpredicts the observed value for $\sim 80 \%$ of the targets, and $\left\langle S_{\text {obs }} / S_{\text {pred }}\right\rangle=0.75$. As a result, many of the 


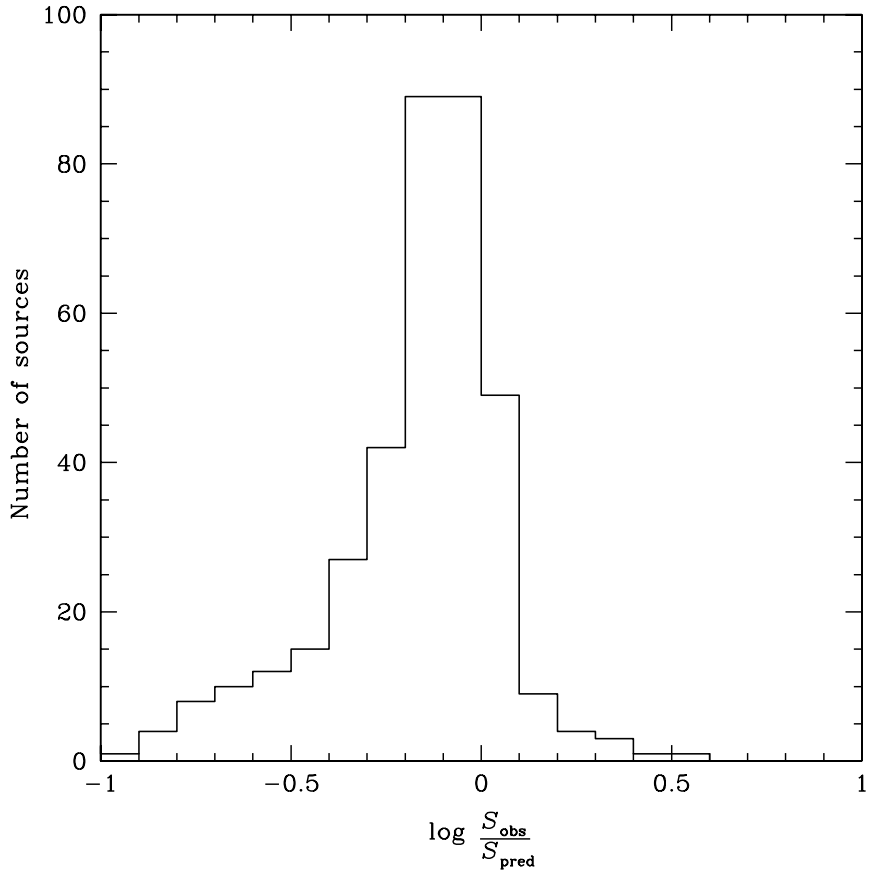

Figure 2. Comparison of the observed values of the $4.85 \mathrm{GHz}$ flux densities to the predicted values based on power-law extrapolation from archival data at $\sim 0.35 \mathrm{GHz}$ and $1.4 \mathrm{GHz}$.

observed sources do not end up satisfying the nominal CRATES cuts on flux density $\left(S_{4.8 \mathrm{GHz}} \geqslant 65 \mathrm{mJy}\right)$ and/or spectral index $(\alpha>-0.5)$. Indeed, by requiring a bright detection at $\sim 0.35 \mathrm{GHz}$, we seem to have selected a significant number of gigahertz-peaked spectrum (GPS) sources rather than strong flat-spectrum $4.85 \mathrm{GHz}$ sources. Figure 3 shows a scatter plot of the spectral index between $1.4 \mathrm{GHz}$ and $4.85 \mathrm{GHz}$ (" $\alpha_{\mathrm{NVSS} / \mathrm{Eff}}$ ") versus the spectral index between $\sim 0.35 \mathrm{GHz}$ and $1.4 \mathrm{GHz}$ (" $\alpha$ low/NVSS"). The shaded region represents sources with spectra that rise from $\sim 0.35 \mathrm{GHz}$ to $1.4 \mathrm{GHz}$ but fall from $1.4 \mathrm{GHz}$ to $4.85 \mathrm{GHz}$ (i.e., GPS sources); our catalog contains 75 such sources. Note also that most sources $(>80 \%)$ fall below the dashed line, indicating spectra that are steeper above $1.4 \mathrm{GHz}$ than below it. An alternative approach to sample selection, such as simply targeting the brightest NVSS sources, may have yielded a higher fraction of CRATES-like sources.

Nevertheless, the results of this survey are of value. Of the $\sim 160$ CRATES-like sources missing in the north polar cap, we have identified 57 , bringing the coverage there from $\sim 33 \%$ to $\sim 57 \%$. Of the $\sim 100$ missing sources in the PMN holes, we have identified 24 , bringing the coverage there from $0 \%$ to $\sim 24 \%$. The total coverage of the $|b|>10^{\circ}$ sky is increased from $97.6 \%$ to $98.3 \%$. This catalog of strong, compact sources with subarcsecond astrometry is also of great use for calibrating radio telescope arrays. Additionally, our $4.85 \mathrm{GHz}$ survey, along with the $8.4 \mathrm{GHz}$ follow-up measurements, brings the fraction of high-latitude WMAP point sources with CRATESlike counterparts to $84 \%$. Our observations will be even more important in the Planck era; the increase in sensitivity $(\sim 30 \times)$ over WMAP will yield thousands of point sources over the entire sky, and the identification of counterparts will rely heavily on catalogs of bright, flat-spectrum radio sources.

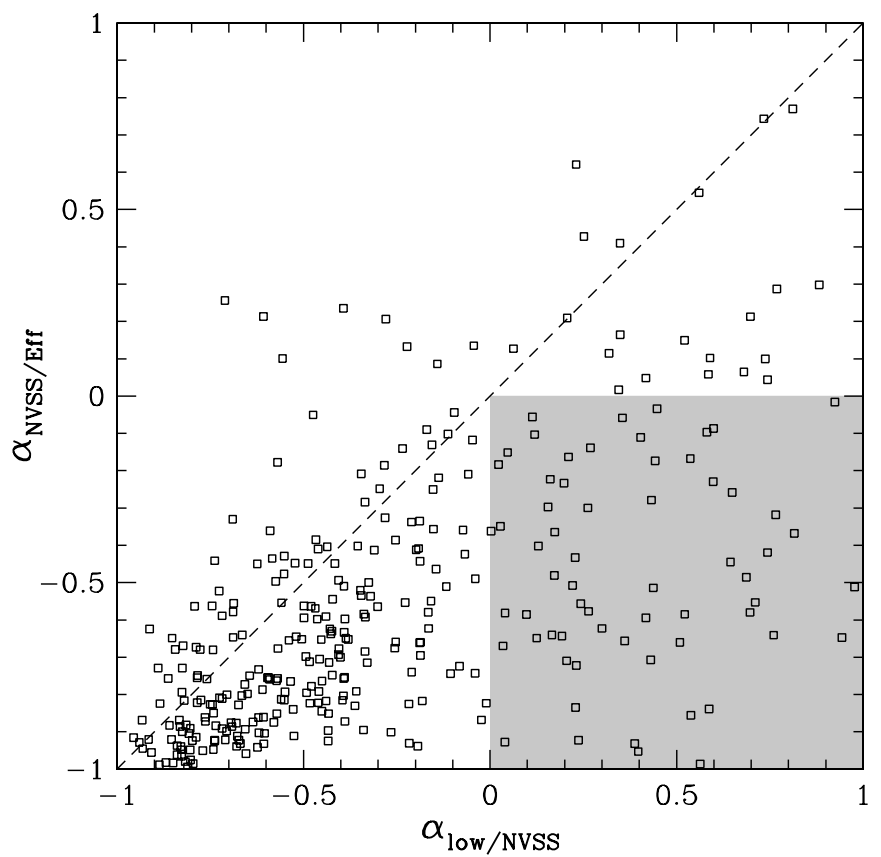

Figure 3. Comparison of spectral indices from $1.4 \mathrm{GHz}$ to $4.85 \mathrm{GHz}$

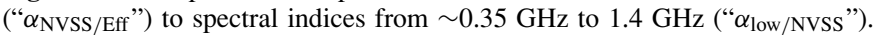
Gigahertz-peaked spectrum (GPS) sources lie in the shaded region. Sources below the dashed line are steeper above $1.4 \mathrm{GHz}$ than below it.

Data in this paper are based on observations with the $100 \mathrm{~m}$ telescope of the MPIfR (Max-Planck-Institut für Radioastronomie) at Effelsberg. The National Radio Astronomy Observatory is operated by Associated Universities, Inc., under cooperative agreement with the National Science Foundation. S.E.H. was supported by SLAC under DOE contract DE-AC03$76 \mathrm{SF} 00515$.

\section{REFERENCES}

Abdo, A. A., et al. 2009, ApJ, 700, 597

Angelakis, E., Kraus, A., Readhead, A. C. S., Zensus, J. A., Bustos, R., Krichbaum, T. P., Witzel, A., \& Pearson, T. J. 2009, A\&A, 501, 801

Baars, J. W. M., Genzel, R., Pauliny-Toth, I. I. K., \& Witzel, A. 1977, A\&A 61,99

Browne, I. W. A., et al. 2003, MNRAS, 341, 13

Condon, J. J., Cotton, W. D., Greisen, E. W., Yin, Q. F., Perley, R. A., Taylor, G. B., \& Broderick, J. J. 1998, AJ, 115, 1693

Douglas, J. N., Bash, F. N., Bozyan, F. A., Torrence, G. W., \& Wolfe, C. 1996, AJ, 111,1945

Gregory, P. C., Scott, W. K., Douglas, K., \& Condon, J. J. 1996, ApJS, 103, 427

Griffith, M. R., \& Wright, A. E. 1993, AJ, 105, 1666

Hartman, R. C., et al. 1999, ApJS, 123, 79

Healey, S. E., Romani, R. W., Taylor, G. B., Sadler, E. M., Ricci, R., Murphy, T., Ulvestad, J. S., \& Winn, J. N. 2007, ApJS, 171, 61

Healey, S. E., et al. 2008, ApJS, 175, 97

Hinshaw, G., et al. 2007, ApJS, 170, 288

Kühr, H., Pauliny-Toth, I. I. K., Witzel, A., \& Schmidt, J. 1981, AJ, 86, 854

Myers, S. T., et al. 2003, MNRAS, 341, 1

Ott, M., Witzel, A., Quirrenbach, A., Krichbaum, T. P., Standke, K. J., Schalinski, C. J., \& Hummel, C. A. 1994, A\&A, 284, 331

Rengelink, R. B., Tang, Y., de Bruyn, A. G., Miley, G. K., Bremer, M. N., Röttgering, H. J. A., \& Bremer, M. A. R. 1997, A\&AS, 124, 259

Sowards-Emmerd, D., Romani, R. W., \& Michelson, P. F. 2003, ApJ, 590, 109 Wright, E. L., et al. 2008, ApJS, 180, 283 